\title{
Circadian rhythm characteristics of oral squamous cell carcinoma growth in an orthotopic xenograft model
}

This article was published in the following Dove Press journal:

OncoTargets and Therapy

22 January 2013

Number of times this article has been viewed

\author{
Ningbo Zhao* \\ Hong Tang* \\ Kai Yang \\ Dan Chen
}

Department of Oral and Maxillofacial Surgery, the First Affiliated Hospital of Chongqing Medical University, Chongqing, China

*These authors contributed equally to this work
Correspondence: Kai Yang

Department of Oral and Maxillofacial Surgery, the First Affiliated Hospital of Chongqing Medical University, No I, Youyi Road, Yuzhong District, Chongqing 400016, China

Tel +86 23 890I 2569

$\mathrm{Fax}+862389012569$

Email cqfyyk@hotmail.com
Background: Recent studies show that circadian rhythm changes are closely related to the occurrence and development of various tumors, such as breast, liver, and prostate. However, there are significant differences in circadian rhythm between different tumors. At present, the circadian rhythm characteristics of oral cancer remain unknown. The purpose of this study is to investigate the circadian rhythm characteristics of the in vivo growth of oral squamous cell carcinoma (OSCC).

Materials and methods: Thirty-two nude mice were placed under 12-hour light/12-hour dark cycles. The human OSCC cell line BcaCD885 was inoculated in the cheek of nude mice. After 3 weeks, eight mice were sacrificed at four time points, including 4 hours after light onset (HALO), 10 HALO, 16 HALO, and 22 HALO, during a period of 24 hours. The volume of excised tumors was measured and the proliferative index (PI) and apoptotic index (AI) of tumor cells were determined by flow cytometry. A cosine analysis method was used to determine whether the tumor volume, PI, and AI obeyed a circadian rhythm.

Results: There was a significant circadian rhythm in the tumor volume and PI of OSCC cells. For the tumor volume, there were significant differences between the four time points. The peak and trough values of the tumor volume appeared at 3.23 HALO and 15.23 HALO, whereas the peak and trough values of PI appeared at 6.60 HALO and 18.16 HALO, respectively. However, there was no circadian rhythm in the AI of tumor cells, despite significant differences between the four time points.

Conclusion: This study demonstrates, for the first time, that the tumor volume and PI of in vivo growing OSCC undergo circadian rhythms. These results support the assertion that time factor should be considered in the occurrence, development, treatment, efficacy evaluation and pathophysiology of OSCC.

Keywords: oral carcinoma, proliferation, apoptosis

\section{Introduction}

Along with the earth's rotation and alternating circadian rhythms, many of the physiological activities of the human body, such as body temperature, heart rate, blood pressure, hormone secretion, and cellular metabolism, undergo circadian rhythms. ${ }^{1,2}$ The circadian rhythms cause obvious biological rhythms of cellular metabolism and proliferation in the rest and activity phases of humans. ${ }^{1,3,4}$ Circadian rhythms are not only present in the normal human body and cells, but have also been shown to influence the proliferation of tumor cells. ${ }^{1-7}$ Malignant tumors show an uncontrolled and disordered cell proliferation, which is necessarily reflected in the time dimension. ${ }^{3}$ Changes in circadian rhythms have been shown to be closely related to the development 
of tumors, the proliferation of tumor cells, and therapeutic effects and side effects of anticancer drugs, ${ }^{2-8}$ and to be differentially involved in the growth of different tumor cell types. ${ }^{9,10}$ Hence, the investigation of tumor circadian rhythms will provide new ideas and methods for the development of individualized treatments.

The suprachiasmatic nucleus in the supraoptic decussation of the hypothalamus has been reported to be the controller of circadian rhythms in mammals. ${ }^{3}$ The suprachiasmatic nucleus controls the rest-activity pattern by regulating circadian rhythms with the light changes during day and night. Circadian rhythms affect tissues and cells through neurotransmitters and endocrine regulation, controlling all levels of life processes, such as activity, rest, metabolism, cell proliferation, endocrine function, and immune responses, in a coordinated and timely ordered manner. ${ }^{7,11}$ Therefore, circadian rhythm time is expressed by hours after light onset (HALO) rather than by clock time. It has been confirmed that at least nine core circadian genes, period 1 (Per 1$)$, period 2 (Per2), period 3 (Per3), circadian locomotor output cycles kaput (Clock), cryptochrome 1 (Cryl), cryptochrome 2 (Cry2), brain and muscle Arnt-like protein 1 (Bmal 1), casein kinase $1 \varepsilon(C K 1 \varepsilon)$, and timeless (Tim), participate in the regulation of mammalian circadian rhythms. ${ }^{1,4,8}$ Since there are significant individual differences in the biological rhythms in different organisms, they are prone to interference from various factors. The cosine analysis method, developed from the cosine function model, is an effective method for the analysis of biological rhythms. The cosine analysis method, through cosine curve fitting, can exclude the interference of other biological factors, isolate the biological rhythm under study, and intuitively show its characteristics. ${ }^{12,13}$

Rodents are commonly used animal models in the study of biological rhythms. ${ }^{3,7,13-15}$ Although rodents are nocturnal animals and show reverse rest and activity phases compared with humans, in vivo studies of the changes in the circadian rhythms of cellular metabolism and responses to anticancer drugs have demonstrated that the experimental results obtained from the rest and activity phases of rodents can be extrapolated to the corresponding rest and activity phases of humans..$^{15,16}$

Oral cancer accounts for about $2 \%$ of systemic malignancies. Approximately 274,000 new cases of oral carcinoma have been diagnosed all over the world every year. ${ }^{17,18}$ Ninety percent of the oral cancers correspond to squamous cell carcinomas. Although great progress has been achieved in the development of various therapeutic methods for oral carcinoma in recent decades, the 5-year overall survival rate for oral cancer patients after treatment is only approximately $55 \%-60 \% .{ }^{17-19}$ Therefore, it is of great significance to identify the mechanisms underlying oral cancer prevention and to explore new and more effective therapeutic options. To date, no study on the circadian rhythm characteristics of oral cancer growth in vivo has been reported. In this study, the human oral squamous cell carcinoma (OSCC) cell line BcaCD885 was inoculated in the cheek of nude mice, in order to establish an OSCC mouse model..$^{20,21}$ Three of the most representative indicators of tumor growth (tumor volume, cell proliferation, and apoptosis) were chosen to reflect the circadian rhythm characteristics of OSCC during a 24-hour period. We hope to provide new ideas for the study of individualized OSCC treatments that have the circadian rhythm of tumor growth taken into consideration.

\section{Materials and methods Instruments and reagents}

The human OSCC cell line BcaCD885 was obtained from the College of Life Sciences of Chongqing Medical University. Flow cytometry (FCM) was performed using a FACScan flow cytometer (BD Biosciences, Franklin Lakes, NJ, USA). Frozen low-speed centrifuge (Z233MK-2) was purchased from Hermle Labortecknik $\mathrm{GmbH}$ (Wehingen, Germany). The light microscope was a Leica ts-26 (Leica Microsystems, Wetzlar, Germany).

\section{Animals}

Specific pathogen-free (SPF) Balb/c nude mice (male, 5 6 weeks, 18 20 g) were purchased from the Experimental Animal Center of the Chongqing Medical University. Mice were placed in constant temperature and humidity conditions. The bedding, food, and water for the mice were all sterilized. All procedures were performed in accordance with the guidelines of the Committee on Animals of the Chongqing Medical University.

\section{Establishment of a nude mouse model of oral squamous cell carcinoma}

Thirty-two nude mice were randomly housed in separate cages (four mice per cage). Before being used in studies, mice were housed for 3 weeks under 12-hour light/12-hour dark cycles (at a room temperature of $24^{\circ} \mathrm{C} \pm 1{ }^{\circ} \mathrm{C}$ and humidity of $60 \% \pm 10 \%$ ). In the 12-hour light/dark cycles, the number of hours after light onset (HALO) was taken as a reference for time. Thus, " 0 HALO" was set as the time to turn on the light and "12 HALO" as the time to turn off the light. After 3 weeks, BcaCD885 cells growing at exponential 
phase were trypsinized with $0.5 \%$ of trypsin, centrifuged for 4 minutes $\left(800 \mathrm{rpm}, 4^{\circ} \mathrm{C}\right)$, and resuspended in sterile phosphate-buffered saline (PBS). Then, $0.2 \mathrm{~mL}$ of PBS containing $2 \times 10^{6} \mathrm{BcaCD} 885$ cells were injected under the oral mucosa of nude mice to establish a nude mouse model of OSCC. Afterwards, mice continued to be housed under 12-hour light/dark cycles. After 3 weeks, when noticeable tumors were present, mice from two cages (eight mice) were randomly sacrificed, by cervical dislocation, at four time points, including 4 HALO, 10 HALO, 16 HALO, and 22 HALO, during a period of 24 hours, to a total of 32 mice. All tumors were excised immediately after sacrifice.

\section{Calculation of tumor volume}

Excised tumors were rinsed with PBS and dried on filter paper. Tumors were measured with a caliper rule and tumor volume was calculated by the following formula:

$$
\left(\text { Width }^{2} \times \text { Length }\right) / 2 \text {. }
$$

\section{Cell and histology examination of tumor tissues of BcaCD885}

Each one of the 32 freshly excised tumor tissues was equally divided into two parts. One part was prepared to evaluate cell proliferation and apoptotic index, as mentioned below, while the other part was embedded in paraffin and cut into $4 \mu \mathrm{m}$ thick sections and stained by hematoxylin and eosin (HE). Tumor cells of HE stained sections were observed under light microscope.

\section{Proliferation and apoptosis assays}

The above-prepared 32 pieces of freshly excised tumor tissues were disaggregated in order to prepare tumor cell suspensions. Proliferation and apoptosis of tumor cells were examined by FCM. Briefly, the necrotic tissues surrounding the tumors were first removed. After washing three times with D-Hanks solution, tumors were immersed for 20 minutes in a solution containing penicillin $\left(5 \times 10^{5} \mathrm{U} / \mathrm{L}\right)$ and streptomycin $(100 \mathrm{mg} / \mathrm{L})$. Then, tumors were cut into small pieces $\left(\sim 1 \mathrm{~mm}^{3}\right)$ and immersed in a tissue-digestive solution $(2 \mathrm{~g} / \mathrm{L}$ of collagenase, $1 \mathrm{~g} / \mathrm{L}$ of deoxyribonuclease [DNase] and $0.0002 \mathrm{~g} / \mathrm{L}$ of hyaluronidase) for 40 minutes. Upon filtration with steel net (200-mesh), the filtrate was centrifuged $(1000 \mathrm{rpm} \times 5$ minutes $)$, and the supernatant was discarded. After washing three times with PBS, the cell pellet was resuspended in RPMI-160 medium, and the tumor cell suspension was processed for proliferation and apoptosis analysis.
The cell proliferation assay was performed as described below. Briefly, cell concentration was adjusted to $1 \times 10^{6}$ cells $/ \mathrm{mL}$. One milliliter of tumor cell suspension was centrifuged $\left(1000 \mathrm{rpm} \times 5\right.$ minutes, $\left.4^{\circ} \mathrm{C}\right)$ and the supernatant was discarded. Cells were fixed with $70 \%$ ethanol at $-20^{\circ} \mathrm{C}$ and incubated at $4^{\circ} \mathrm{C}$ overnight. After centrifugation $\left(1000 \mathrm{rpm} \times 5\right.$ minutes, $\left.4^{\circ} \mathrm{C}\right)$, cells were washed twice with PBS. One milliliter of propidium iodide staining solution was added. After a 30-minute incubation at $4^{\circ} \mathrm{C}$ in the dark, the cell suspension was analyzed by FCM. The following formula was used to calculate the proliferation index (PI) of the tumor cells:

$$
\mathrm{PI}=(\mathrm{S}+\mathrm{G} 2 / \mathrm{M}) /(\mathrm{G} 0 / \mathrm{G} 1+\mathrm{S}+\mathrm{G} 2 / \mathrm{M}) \times 100 \%,
$$

where G0, G1, G2, S, and M correspond to the different phases of the cell cycle.

The cell apoptosis assay was performed as described below. Briefly, cell concentration was adjusted to $1 \times 10^{6}$ cells $/ \mathrm{mL}$. One milliliter of tumor cell suspension was centrifuged $\left(1000 \mathrm{rpm} \times 5\right.$ minutes, $\left.4^{\circ} \mathrm{C}\right)$, and the supernatant was discarded. Cells were washed twice with PBS at $4{ }^{\circ} \mathrm{C}$ and incubated with $200 \mu \mathrm{L}$ of Annexin V staining solution for 15 minutes at room temperature. Afterwards, $1 \mathrm{~mL}$ of PI staining solution was added, and after 5 minutes, the cell suspension was analyzed by FCM. The following formula was used to calculate the apoptotic index (AI) of the tumor cells:

$$
\begin{aligned}
\mathrm{AI}= & (\text { Number of apoptotic cells/Total number } \\
& \text { of cells }) \times 100 \% .
\end{aligned}
$$

\section{Statistical analysis}

All data in this study were processed using SPSS 13.0 software (IBM Corporation, Armonk, NY, USA). One-way analysis of variance (ANOVA) was used to analyze the significance of the differences in the indicators of tumor growth between the four time points during a 24 hour light/dark cycle period. The CircWave 1.4 software (Hut RA, Groningen, The Netherlands) was used to perform cosine analysis, so as to determine whether the indicators of tumor growth undergo a circadian rhythm. Data were expressed as mean \pm standard deviation (SD). A $P$-value $<0.05$ was considered statistically significant.

\section{Results \\ Cell and histology examination of tumor tissues of BcaCD885}

Three weeks after BcaCD885 cells were injected under the cheek mucosa of 32 nude mice, all mice had noticeable 
tumors in the cheek. HE stained sections of tumor tissues were observed under light microscope. A large number of poorly differentiated OSCC cells were tightly arranged and a cancer nest existed (Figure 1).

\section{Variation of tumor volume obeys a circadian rhythm}

The OSCC tumor volume at different time points during a 24-hour period is depicted in Table 1. Cosine analysis showed that the variation of tumor volume conformed to the law of cosines. A single cosine test confirmed that the variation of tumor volume obeyed a circadian rhythm $(\mathrm{F}=37.91$, $P=0.00)$. In accordance with the correspondent cosine curve fitting, shown in Figure 2A, the peak value of the tumor volume appeared at $3.23 \mathrm{HALO}$ (the corresponding volume was $477.48 \mathrm{~mm}^{3}$ ), whereas the trough value appeared at 15.23 HALO (the corresponding volume was $342.05 \mathrm{~mm}^{3}$ ). ANOVA results indicated that there were significant differences of the tumor volume between the different time points $(\mathrm{F}=52.26, P=0.00)$ : 4 HALO versus $10 \mathrm{HALO}$ $(t=4.67, P<0.01)$; 4 HALO versus 16 HALO $(t=7.31$, $P<0.01)$; 4 HALO versus $22 \mathrm{HALO}(t=3.57, P<0.01)$; 10 HALO versus $16 \mathrm{HALO}(t=11.98, P<0.01)$; $10 \mathrm{HALO}$ versus $22 \mathrm{HALO}(t=8.24, P<0.01)$; and 16 HALO versus $22 \operatorname{HALO}(t=3.74, P<0.01)$.

\section{Variation of tumor cell PI obeys a circadian rhythm}

The PI of OSCC at different time points during a 24-hour period is depicted in Table 1 . Cosine analysis showed that

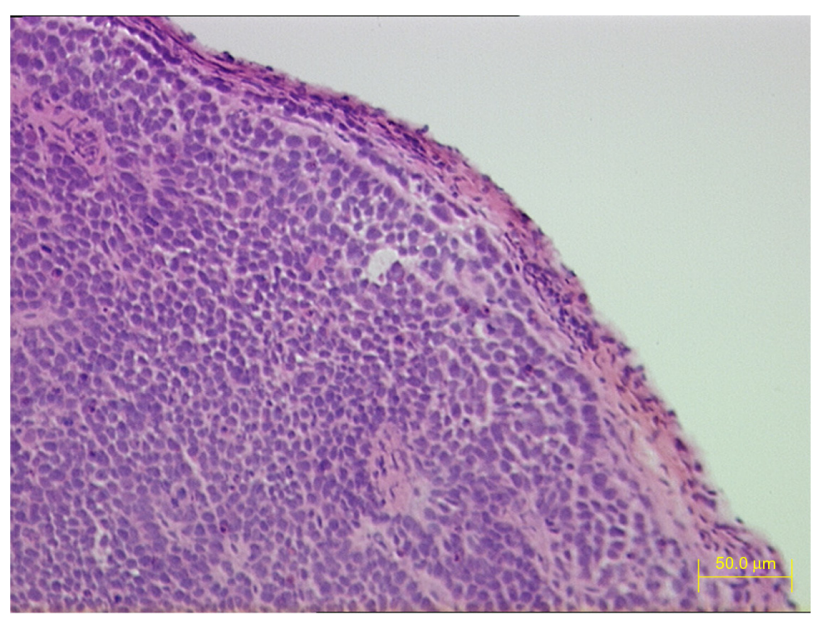

Figure I HE staining of tumor tissue sections 3 weeks after BcaCD885 cells were inoculated under the cheek mucosa of nude mice.

Note: $\mathrm{HE} \times 200$.

Abbreviation: $\mathrm{HE}$, hematoxylin and eosin. the variation in the PI of tumor cells conformed to the law of cosines. A single cosine test confirmed that the variation of the tumor cell PI obeyed a circadian rhythm $(\mathrm{F}=9.71$, $P=0.00)$. In accordance with the correspondent cosine curve fitting, shown in Figure 2B, the peak value of PI appeared at 6.60 HALO (the corresponding PI was 43.39), whereas the trough value of PI appeared at 18.16 HALO (the corresponding PI was 35.31). ANOVA results indicated that there was no significant difference in the PI between the different time points $(\mathrm{F}=1.59, P=0.27)$.

\section{Variation of tumor cell Al does not obey a circadian rhythm}

The AI of OSCC at different time points during a 24-hour period is depicted in Table 1 . Cosine analysis showed that the variation in the tumor cells AI did not obey a circadian rhythm, which was demonstrated by a single cosine test. Nevertheless, ANOVA results indicated that there were significant differences in the tumor cell AI between the different time points $(\mathrm{F}=10.561, P=0.004)$ : 4 HALO versus $10 \mathrm{HALO}(t=4.938, P<0.01)$; 10 HALO versus 16 HALO $(t=4.537, P<0.01)$, but $10 \mathrm{HALO}$ versus $22 \mathrm{HALO}$ $(t=2.125, P>0.05) ; 16$ HALO versus $22 \mathrm{HALO}(t=2.412$, $P>0.05) ; 4$ HALO versus $22 \mathrm{HALO}(t=2.813, P>0.05)$ and 4 HALO versus $16 \mathrm{HALO}(t=0.401, P>0.05)$.

\section{Discussion}

To date, it has been confirmed that circadian rhythm changes are closely related to the occurrence, development, response to therapy, and the prognosis of a number of cancers, such as breast, liver, prostate, endometrial, colon, and non-small cell lung cancer. ${ }^{2-8}$ There are significant differences in circadian rhythm between different tumor cells. ${ }^{9,10}$ Recently, Sato et al reported that PER 1 and PER3 exhibit, respectively, a high and low expression in oral gum cancer cells. ${ }^{22}$ Upon cisplatin treatment, PER 1 and PER3 played, respectively, anti- and proapoptotic roles in CA9-22 gum cancer cells that were cultured in vitro. Studies by Bjarnason et $\mathrm{al}^{23}$ and by Zieker et $\mathrm{al}^{24}$ showed that PER 1 expression had circadian rhythm characteristics during a period of 24 hours in healthy human oral mucosa cells. The level of PER1 expression could significantly influence the expressions of cell cycle related proteins and tumor-related genes, such as cyclin $\mathrm{E}$, cyclin B, cyclin A, p53, c-Myc, and so on. Gery et al ${ }^{25}$ studied PER 1 expression in the human colon cancer cell line HCT116 in vitro and found that PERl regulated cell cycle related protein expressions, such as wee-1, cyclin B1, Cdc2, and so on, resulting in influences on tumor genesis and 
Table I Changes of OSCC tumor volume, cell proliferative index, and apoptotic index at different time points during a 24-hour period

\begin{tabular}{|c|c|c|c|c|}
\hline & \multicolumn{4}{|c|}{ Time of sacrifice } \\
\hline & 4 HALO & 10 HALO & 16 HALO & 22 HALO \\
\hline Tumor volume & $428.89 \pm 23.78$ & $486.24 \pm 37.05$ & $339.01 \pm 30.57$ & $384.93 \pm 40.71$ \\
\hline PI & $42.72 \pm 8.04$ & $41.67 \pm 4.27$ & $36.46 \pm 0.39$ & $36.55 \pm 0.29$ \\
\hline $\mathrm{Al}$ & $24.60 \pm 1.49$ & $47.18 \pm 1.80$ & $26.43 \pm 10.80$ & $37.46 \pm 1.82$ \\
\hline
\end{tabular}

Note: Data are expressed as mean \pm SD

Abbreviations: OSCC, (human) oral squamous cell carcinoma; HALO, hours after light onset; PI, proliferative index; Al, apoptotic index; SD, standard deviation.

cancer development. The basic characteristic of a malignant tumor is uncontrolled growth, proliferation, and apoptosis of cells. However, to date, no study about the circadian rhythm of in vivo oral cancer growth has been reported. In this study, the three most representative indicators (tumor volume, cell proliferation, and apoptosis) of tumor growth were chosen to reflect the circadian rhythm characteristics of OSCC growth in vivo during a 24-hour period. Our results indicated that OSCC tumor volume at different time points obeyed a 24-hour cycle circadian rhythm. The circadian variation of tumor volume had a peak value of $477.48 \mathrm{~mm}^{3}$ at $3.23 \mathrm{HALO}$ and trough value of $342.05 \mathrm{~mm}^{3}$ at $15.23 \mathrm{HALO}$, ie, the tumor volume at an early rest phase (3.23 HALO) was increased by $40 \%$ when compared with that at an early activity phase (15.23 HALO). Cell proliferation analysis showed that changes of OSCC PI also obeyed a 24-hour cycle circadian rhythm. The circadian rhythm of PI had a peak value of $43.39 \mathrm{~mm}^{3}$ at $6.60 \mathrm{HALO}$ and trough value of $35.31 \mathrm{~mm}^{3}$ at $18.16 \mathrm{HALO}$, ie, the PI at a middle rest phase ( 6.60 HALO) increased by $23 \%$ when compared with that at a middle activity phase (18.16 HALO). Although the

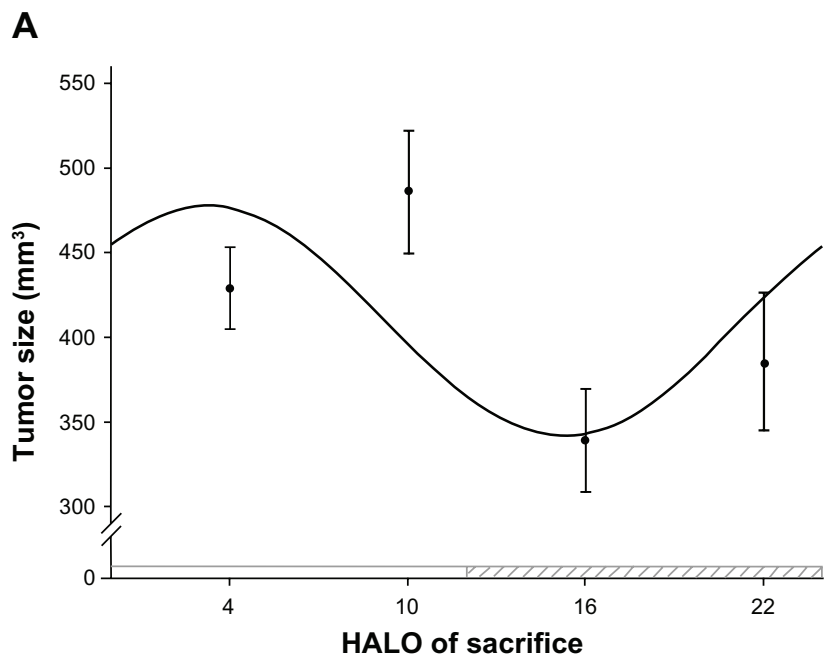

analysis of apoptosis showed that variations in OSCC AI did not obey a 24-hour cycle circadian rhythm, there were significant differences between different time points. We assumed that the above differences may be due to the expression of Clock gene. PERl in oral cancer cells had circadian rhythm during a period of 24 hours. PER 1 regulates cell cycle related proteins and tumor related genes, ${ }^{23-25}$ which leads to circadian variations in tumor size and PI of OSCC growth in vivo. Nevertheless, the specific mechanism still needs further and more extensive research. Our study investigated, for the first time, the circadian rhythm of oral cancer growth in vivo. Although this needs to be explored further, our results indicate that the time factor should be considered in the individualized treatment of OSCC. For example, the evaluation of the efficacy of various anticancer agents should be performed at the same time points during the day. We assumed that a higher therapeutic efficacy would be achieved if the anticancer drugs are administered in the middle rest phase, in which the cell proliferation level of OSCC is the highest during a day. Similarly, if the surgical time is in the early or middle activity phase, in which the

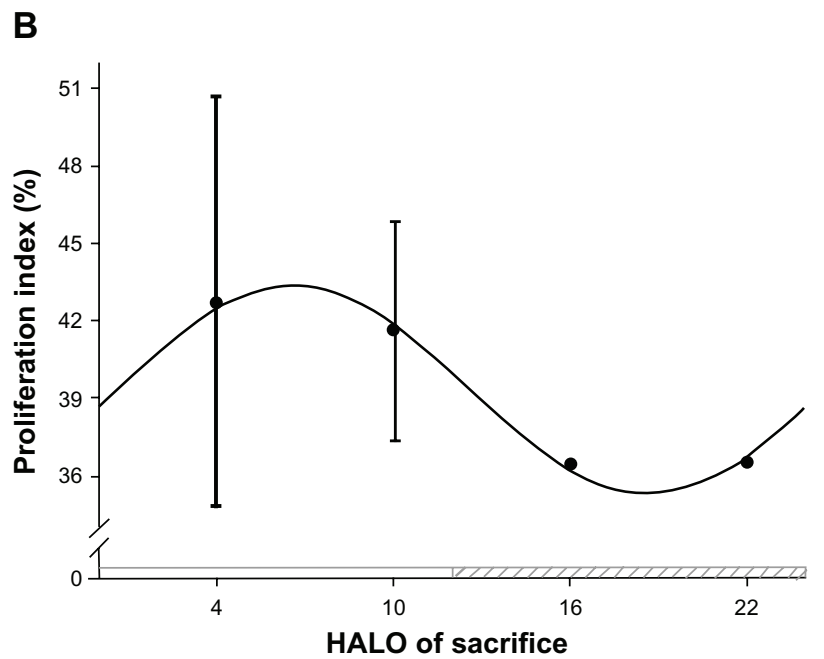

Figure 2 Circadian rhythm of OSCC tumor growth in vivo. (A) Cosine fitted curve of OSCC tumor volume at different time points during a 24-hour period; (B) cosine fitted curve of $\mathrm{PI}$ of tumor cells at different time points during a 24-hour period.

Abbreviations: HALO, hours after light onset; OSCC, (human) oral squamous cell carcinoma; PI, proliferative index. 
tumor volume and cell proliferation level of OSCC are the lowest during a day, tumor recurrence and surgical trauma might be reduced.

This study provides the basis for further investigation into the molecular mechanisms underlying the circadian rhythm of in vivo OSCC growth. We believe that an in-depth study of the relation and mechanism of circadian rhythm and tumor growth will be reflected into a new understanding of the occurrence and development of malignant tumors and in the design of new molecular therapeutic targets. Also, the time factor should be taken into consideration in the efficacy evaluation of radiotherapy, chemotherapy, and surgery, resulting in a new model of tumor prevention and therapy.

\section{Disclosure}

The authors report no conflicts of interest in this work.

\section{References}

1. Eismann EA, Lush E, Sephton SE. Circadian effects in cancer-relevant psychoneuroendocrine and immune pathways. Psychoneuroendocrinology. 2010;35(7):963-976.

2. Smolensky MH, Peppas NA. Chronobiology, drug delivery, and chronotherapeutics. Adv Drug Deliv Rev. 2007;59(9-10):828-851.

3. Murphy PJ, Campbell SS. Physiology of the circadian system in animals and humans. J Clin Neurophysiol. 1996;13(1):2-16.

4. Huang XL, Fu CJ, Bu RF. Role of circadian clocks in the development and therapeutics of cancer. J Int Med Res. 2011;39(6):2061-2066.

5. Lemmer B. Chronobiology, drug-delivery, and chronotherapeutics. $A d v$ Drug Deliv Rev. 2007;59(9-10):825-827.

6. Lévi F. Circadian chronotherapy for human cancers. Lancet Oncol. 2001;2(5):307-315.

7. Jasser SA, Blask DE, Brainard GC. Light during darkness and cancer: relationships in circadian photoreception and tumor biology. Cancer Causes Control. 2006;17(4):515-523.

8. Fu L, Lee CC. The circadian clock: pacemaker and tumour suppressor. Nat Rev Cancer. 2003;3(5):350-361.

9. Klevecz RR, Braly PS. Circadian and ultradian cytokinetic rhythms of spontaneous human cancer. Ann N Y Acad Sci. 1991;618:257-276.

10. Sephton S, Spiegel D. Circadian disruption in cancer: a neuroendocrineimmune pathway from stress to disease? Brain Behav Immun. 2003;17(5):321-328.
11. Filipski E, Lévi F. Circadian disruption in experimental cancer processes. Integr Cancer Ther. 2009;8(4):298-302.

12. Manoharan S, Baskar AA, Manivasagam T, Subramanian P. Circadian rhythmicity of plasma lipid peroxidation and antioxidants in oral squamous cell carcinoma. Singapore Med J. 2005;46(4):184-188.

13. Manivasagam T, Subramanian P. Monosodium glutamate affects the temporal characteristics of biochemical variables in Wistar rats. Pol $J$ Pharmacol. 2004;56(1):79-84.

14. Lévi F, Okyar A, Dulong S, Innominato PF, Clairambault J. Circadian timing in cancer treatments. Annu Rev Pharmacol Toxicol. 2010;50: $377-421$.

15. Innominato PF, Lévi FA, Bjarnason GA. Chronotherapy and the molecular clock: Clinical implications in oncology. Adv Drug Deliv Rev. 2010;62(9-10):979-1001.

16. Lévi F. Chronotherapeutics: the relevance of timing in cancer therapy. Cancer Causes Control. 2006;17(4):611-621.

17. Parkin DM, Bray F, Ferlay J, Pisani P. Global cancer statistics, 2002. CA Cancer J Clin. 2005;55(2):74-108.

18. Kademani D, Bell RB, Schmidt BL, et al; the American Association of Oral and Maxillofacial Surgeons Task Force on Oral Cancer. Oral and maxillofacial surgeons treating oral cancer: a preliminary report from the American Association of Oral and Maxillofacial Surgeons Task Force on Oral Cancer. J Oral Maxillofac Surg. 2008;66(10):2151-2157.

19. Kademani D, Bell RB, Bagheri S, et al. Prognostic factors in intraoral squamous cell carcinoma: the influence of histologic grade. $J$ Oral Maxillofac Surg. 2005;63(11):1599-1605.

20. Yang K, Cao YA, Shi C, et al. Quantum dot-based visual in vivo imaging for oral squamous cell carcinoma in mice. Oral Oncol. 2010;46(12): 864-868.

21. Yang K, Zhang FJ, Tang H, et al. In-vivo imaging of oral squamous cell carcinoma by EGFR monoclonal antibody conjugated near-infrared quantum dots in mice. Int J Nanomedicine. 2011;6:1739-1745.

22. Sato F, Wu Y, Bhawal UK, et al. PERIOD1 (PER1) has anti-apoptotic effects, and PER3 has pro-apoptotic effects during cisplatin (CDDP) treatment in human gingival cancer CA9-22 cells. Eur J Cancer. 2011;47(11):1747-1758.

23. Bjarnason GA, Jordan RC, Wood PA, et al. Circadian expression of clock genes in human oral mucosa and skin: association with specific cell-cycle phases. Am J Pathol. 2001;158(5):1793-1801.

24. Zieker D, Jenne I, Koenigsrainer I, et al. Circadian expression of clock- and tumor suppressor genes in human oral mucosa. Cell Physiol Biochem. 2010;26(2):155-166.

25. Gery S, Komatsu N, Baldjyan L, Yu A, Koo D, Koeffler HP. The circadian gene per1 plays an important role in cell growth and DNA damage control in human cancer cells. Mol Cell. 2006;22(3):375-382.
OncoTargets and Therapy

\section{Publish your work in this journal}

OncoTargets and Therapy is an international, peer-reviewed, open access journal focusing on the pathological basis of all cancers, potential targets for therapy and treatment protocols employed to improve the management of cancer patients. The journal also focuses on the impact of management programs and new therapeutic agents and protocols on

Submit your manuscript here: http://www.dovepress.com/oncotargets-and-therapy-journal

\section{Dovepress}

patient perspectives such as quality of life, adherence and satisfaction. The manuscript management system is completely online and includes a very quick and fair peer-review system, which is all easy to use. Visit http://www.dovepress.com/testimonials.php to read real quotes from published authors. 